\title{
Research on Control and Design of Island Direct Drive Wind Generator
}

\author{
Lin Xue* \\ School of North China Electric Power University, Baoding, China \\ *745296080@qq.com
}

Keywords: a new resource, microgrids, large power grid, DFIG.

\begin{abstract}
Wind energy has a prominent advantage as a new resource rich in resources and clean and renewable. Nowadays, wind energy is one of the key new energy sources in the world, but the development rate of wind energy resources in the world is less than $20 \%$. Whether it is a large or small wind power industry, its prospects are very broad. in power system operation, microgrids have two operating modes, one is networked operation and the other is islanding operation. This article mainly studies the islanding operation system of microgrid in the situation of disconnecting from the large power grid. Among them, the control strategy is that the system can the key to stable, safe and reliable operation is therefore very necessary for the study of microgrid control strategies in island mode. in this paper, the DFIG control strategy for island and island voltage imbalance conditions is studied. By studying the pitch angle control and speed control of a direct-drive wind turbine, a stable output power is obtained. The way it works.
\end{abstract}

\section{Background}

As the contradiction between the environment and traditional power grids is becoming more and more inconsistent, raw materials for power production are shifting from traditional non-renewable resources to new ones. Technologies such as wind power generation and solar photovoltaic power generation have received strong support from the state, but how to control these wind power the unit has always been an important problem in the world and reducing the influence of wind curtailment and grid stability is also the focus of research across the world.

Wind turbine is a key functional component in wind power generation system. It will intercept the flowing air and convert some of the kinetic energy it carries into mechanical energy. While determining the output power of the entire system, it directly affects the safety of wind turbine operation., stability and reliability.

Among them, the DFIG of doubly-fed induction wind turbine unit is currently the mainstream model of wind power generation. Therefore, this project mainly focuses on researching the design and control strategy of direct-drive generators in island mode and controlling the output power and conversion by means of pitch angle and rotation speed. Efficiency, to achieve efficient use of wind energy, so the project has great research significance and application value.

\section{Research on Direct Drive Wind Generator}

In islanded doubly-fed wind turbines, the three-phase load is usually in an asymmetrical operating state. This will result in unbalanced three-phase voltage and current of the stator of the DFIG. As a result, the output power quality is reduced and the electromagnetic torque of the DFIG is affected by the fluctuation. In the island operation, we need to ensure that the microgrid voltage and frequency meet the requirements.

\subsection{DFIG Control Strategy}

Double-fed induction wind turbine DFIG is currently the mainstream model of wind power generation. When running in isolated islands, we need to ensure that the voltage and frequency of the microgrid meet the requirements. This paper uses the master-slave control method and uses the battery as a reference power source to ensure the PCC of the microgrid. The voltage and frequency remain stable, in order to ensure the stable operation of the micro-grid, that is, the power balance of 
the micro-grid, so the PV and fans and batteries need to adjust the power output according to the size of the load demand. In the island, the DFIG is still controlled according to the vector control strategy during grid connection. The difference is that when the grid is connected to the grid, the fan will output the maximum power, while islanding may need to limit the power output.

In the traditional control mode, the DFIG operates in the maximum wind energy tracking state, and the rotor speed does not change due to the change of the frequency of the microgrid. In order to obtain the inertia characteristic of the synchronous generator, the virtual inertia control link shown in Fig. 1 is introduced into the DFIG maximum power point tracking (MPPT) module. The system frequency change rate $\frac{d f}{d t}$ is used as the input variable. When the frequency of the microgrid changes, the reference value of the electromagnetic power of the DFIG will also change accordingly. This part of the increased electromagnetic power comes from the kinetic energy released by the rotor.

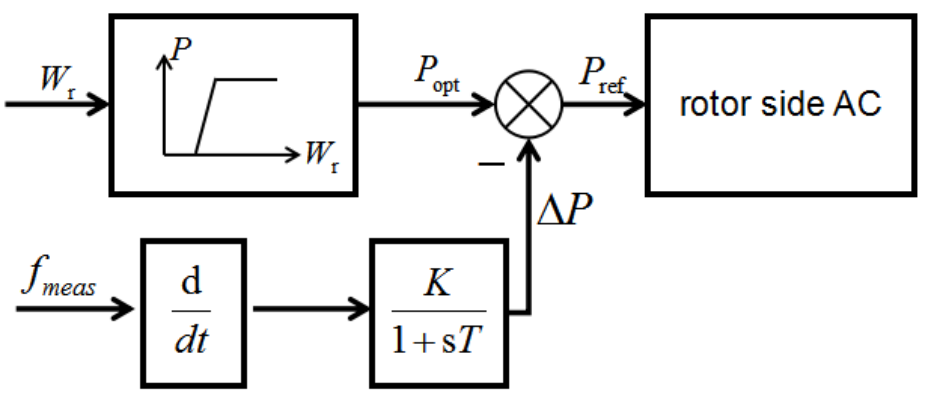

Figure 1. Microgrid frequency adjustment

In the figure above, the choice of $K$ and $T$ values will affect the effect of DFIG's participation in the adjustment of the microgrid frequency. In order to reduce the error and combine the output effect of DFIG, this paper takes the time constant $T$ as $0.1 \mathrm{~s}$. Among them, the moment of inertia $J$ of DFIG is defined as follows:

$$
J=\frac{J_{t u r}}{n^{2}}+J_{g e n}
$$

Where $J_{\text {tur }}$ is the mechanical moment of inertia; $J_{\text {gen }}$ is the electromagnetic moment of inertia; $\mathrm{n}$ is the speed increase ratio of the gear box. The DFIG model's $J_{\text {tur }}$ used in this article is 6100000 $\mathrm{kg} \cdot \mathrm{m}^{2}, J_{\text {gen }}$ is $200 \mathrm{~kg} \cdot \mathrm{m}^{2}$, and $\mathrm{n}$ is 75 .

The DFIG generator is rated at $1500 \mathrm{r} / \mathrm{min}$. By entering the above parameters into equation (1), the DFIG inertia constant $\mathrm{H}$ can be found to be $3.18 \mathrm{~s}$.

After adding the virtual inertia control, the DFIG can simulate the inertia characteristics of the synchronous generator and respond quickly to the frequency change rate $\frac{d f}{d t}$. DFIG's virtual inertial control response speed is fast, although it can only provide short-term frequency support for the microgrid, but it is enough to provide a certain response time for the governors such as diesel engines.

\subsection{Pitch Angle and Speed Control of Output Power}

This section mainly describes the pitch angle control during island operation. At the same time, the speed reference value is set to 1.0. The d-axis speed control on the rotor side will be simpler, and the reference speed is calculated without maximum power tracking.

Islanding operation requires flexible and efficient power adjustments. For doubly-fed reactive wind turbines, the power can be adjusted by pitch angle and rotation speed. For the characteristics of slow response and large vibration of conventional PID pitch system, we propose to control the pitch angle. For linearization, the pitch angle control signal is obtained using active power and speed as input.

Control block diagram as shown below 


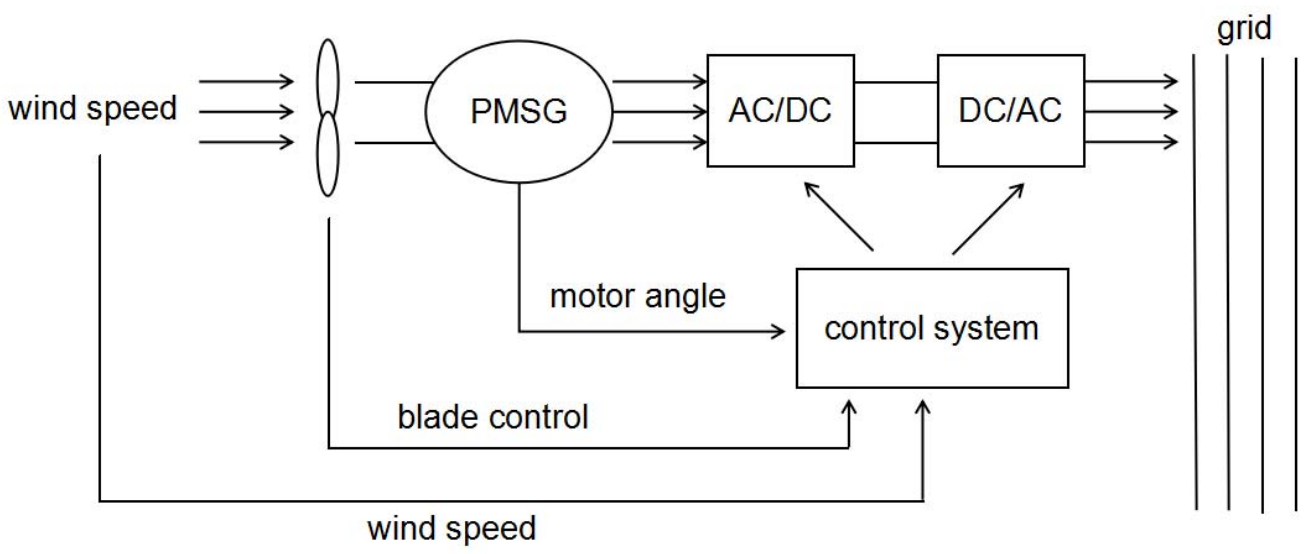

Figure 2. Direct drive wind power system control block diagram

The process of wind power generation system converts wind energy into kinetic energy of the blades, generates torque to drive rotation of the generator rotor, and converts wind energy into mechanical energy. The output power of the fan is a nonlinear process related to the pitch angle of the generator and the wind speed.

Generally, we can calculate the motor speed based on the wind speed and the blade pitch of the wind turbine blades.

$$
\begin{gathered}
\beta_{r e f}=K_{p 1}\left(\omega_{r}-\omega_{r e f}\right)+k_{i 1} \int_{0}^{t}\left(\omega_{r}-\omega_{r e f}\right) d t \\
\frac{\mathrm{d} \beta}{\mathrm{dt}}=\frac{1}{\tau}\left(\beta_{r e f}-\beta\right)
\end{gathered}
$$

Among them: $\omega_{\mathrm{r}}$ is the rotational speed of the generator, and $\omega_{\text {ref }}$ is the reference rotational speed at the current wind speed, $\beta_{\text {ref }}$ is the control target at this moment of wind speed.

The wind energy utilization factor $C_{P}$ is a combination of tip speed ratio $\lambda$ and pitch angle $\beta$, defined as follows:

$$
C_{P}=\frac{P_{0}}{P_{V}}
$$

In the formula, $P_{0}$ is the mechanical power output for the wind turbine and $P_{V}$ is the input power of the wind turbine.

The tip speed ratio is defined as:

$$
\lambda=\frac{R_{W} \omega_{W}}{v}=\frac{R_{W} \omega_{\mathrm{m}}}{N v}
$$

In the above formula, $R_{W}$ is the radius of the wind wheel, $\omega_{W}$ is the mechanical angular speed of the wind turbine, $\omega_{M}$ is the mechanical angular speed of the DFIG, and $N$ is the speed increase ratio of the speed change gear box, $v$ is the wind speed.

Since the generator rotation speed is the rated rotation speed at this time, the tip speed ratio $\lambda$ is determined.

Set the reference value of the generator output active power $P_{S}^{*}$ under the island setting wind speed $V_{W}$. In the pitch angle control, the data fitting method is adopted in this paper. The generator rotation speed is set as the rated rotation speed, and the fan is limited by changing the pitch angle $\beta$. And then the wind energy will be captured.

$$
P_{S}^{*}=\frac{1}{2} C_{P}(\beta, \lambda) \rho \pi S V_{W}^{3}
$$

The formula for the wind energy utilization coefficient is 


$$
\begin{gathered}
C_{P}(\beta, \lambda)=0.5 \mathrm{e}^{-0.17 \lambda}\left(\lambda-0.0022 \beta^{2}-5.6\right) \\
\lambda=\frac{R \cdot 2 \pi f W_{r}}{V_{W} N p}
\end{gathered}
$$

If the reference power is known, the wind speed must be

$$
C_{P}(\beta, \lambda)=P_{S}^{*} / 0.5 \rho S V_{W}^{3}
$$

Ultimately, the wind energy utilization factor $C_{P}$ becomes an amount related to the wind speed $V_{W}$ and the reference power $P_{S}{ }^{*}$.

The control block diagram for adjusting the output power of the generator through the pitch angle when islanding is shown in the figure below.

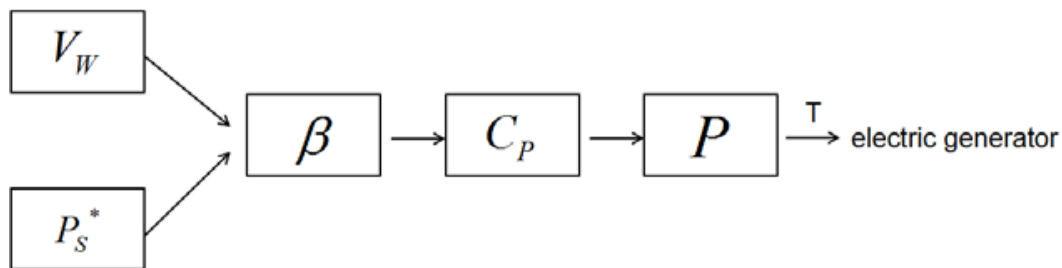

Figure 3. Block diagram of pitch angle control with micgrid operated in islanding mode

\section{Conclusion}

This paper applies the DFIG's virtual inertia control and pitch angle control to the frequency regulation of the microgrid in islanding operation. By controlling the pitch angle, the DFIG is operated in the optimal power tracking mode, which reserves a part of the standby power and is attached to the virtual inertia control link.

The core of this paper is the research on the control strategy of the doubly-fed wind power generation system in the microgrid. The research status of the current wind power generation technology and the double-fed turbine control strategy is mainly studied. The research of the AC-excited variable speed constant-frequency doubly-fed asynchronous wind turbine system is studied. The object, from the generator body, AC excitation converter, microgrid operating state and operating conditions of the wind turbine research, to take the theoretical and practical analysis methods, simulation and other effective ways to conduct in-depth research, academically It is innovative and technically highly practical.

Based on the grid voltage vector control, this paper aims to realize the VSCF and maximum power tracking when the microgrid is connected to the grid. When the island is stable, the double-fed fan can adjust the output power by adjusting the pitch angle.

Micronet is a brand-new topic. It contains a lot of content, such as how to coordinate work among various micro-power supplies to achieve optimal economics, power exchange between micro-grid and large-scale power grid, etc., and study distribution in the micro-grid environment. The power supply has very important practical significance, which also requires us to continue to discover and innovate, and to make our own contribution to the construction of China's smart grid.

\section{References}

[1]. Li Jie. Research on Topology and Control Strategy of Direct-drive Wind Turbine Converter System[D]. Shanghai University, 2009.

[2]. Liu Rui. Micro-grid control strategy based on island model[D]. Shandong University, 2017.

[3]. Chen Lin, Yang Junhua, Chen Sizhe, Wang Qiujing, Wu Jie. Research on Auto-Disturbance Rejection Control for Isolated Island Doubly-Fed Induction Wind Turbine Generator[J]. Renewable Energy,2015,33(02):189-195. 
[4]. Huan Long, Zijun Zhang, Mu-Xia Sun, Yan-Fu Li. The data-driven schedule of wind farm power generations and required reserves[J]. Energy,2018,149. 